\title{
Array-based, Parallel Hierarchical Mesh Refinement Algorithms for Unstructured Meshes
}

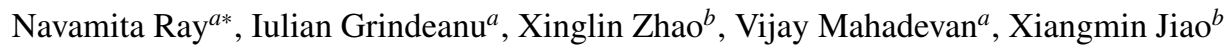 \\ ${ }^{a}$ Mathematics and Computer Science, Argonne National Laboratory, Argonne, IL 60439, USA \\ ${ }^{b}$ Department of Applied Mathematics and Statistics, Stony Brook University, Stony Brook, NY 11794, USA.
}

\begin{abstract}
In this paper, we describe an array-based hierarchical mesh refinement capability through uniform refinement of unstructured meshes for efficient solution of PDE's using finite element methods and multigrid solvers. A multi-degree, multi-dimensional and multi-level framework is designed to generate the nested hierarchies from an initial coarse mesh that can be used for a variety of purposes such as in multigrid solvers/preconditioners, to do solution convergence and verification studies and to improve overall parallel efficiency by decreasing I/O bandwidth requirements (by loading smaller meshes and in-memory refinement). We also describe a high-order boundary reconstruction capability that can be used to project the new points after refinement using high-order approximations instead of linear projection in order to minimize and provide more control on geometrical errors introduced by curved boundaries.

The capability is developed under the parallel unstructured mesh framework "Mesh Oriented dAtaBase" (MOAB [21]). We describe the underlying data structures and algorithms to generate such hierarchies in parallel and present numerical results for computational efficiency and effect on mesh quality. We also present results to demonstrate the applicability of the developed capability to study convergence properties of different point projection schemes for various mesh hierarchies and to a multigrid finite-element solver for elliptic problems.
\end{abstract}

Keywords: uniform mesh refinement, hierarchical meshes, high-order surface reconstruction, parallel computation, half-facet

\section{Introduction}

In the numerical solution of complex partial differential equations (PDE's) using finite element methods for unstructured meshes, the two most computationally intensive steps are mesh generation and linear solvers. An initial coarse mesh representing the computational domain might not be of sufficient resolution to get meaningful results out of the discretizations for physical scales that might be present. As a result, the capability to refine a mesh is an essential part of any simulation process. Additionally, it is well known that multi-level methods such as geometric multigrid methods (GMG)

${ }^{*}$ Corresponding author. Email: nray@mcs.anl.gov. can theoretically deliver optimal time complexity for solving sparse linear systems from PDE discretizations. Thus, it would be advantageous to use nested multilevel (i.e., hierarchical) meshes to achieve high-degree of verifiable accuracy and computational efficiency, especially in the context of large-scale parallel computing, as both the number of processors and the mesh resolution increase. Uniform mesh refinement (UMR) provides a simple and efficient way to generate such hierarchies via successive refinement of the mesh at a previous level. It also provides a natural hierarchy via parent and child type of relationship between entities of meshes at different levels that enable queries that support computation of projection operators between levels. While UMR is a relatively simple process, it is by no means trivial especially in a parallel setting. Notable challenges include maintenance of mesh quality, multi-level and multi-degree refinement, and data structure and software design.

In this paper, we develop parallel uniform refinement- 
based algorithms to generate multi-degree, multidimensional and multi-level meshes from coarse unstructured meshes. The generated mesh hierarchies can be used for a variety of purposes such as convergence studies, multilevel methods, generating large meshes in parallel to overcome IO bottlenecks, etc. While the multi-degree refinement allows achieving uniformly greater resolution faster, the multi-dimensional refinement preserves the hierarchy over explicit lower dimensional entities such as curves in surfaces, or surfaces embedded in volumes.

The key contributions of the paper include: 1) developing a template-based refinement strategy for subdividing each entity into smaller entities to support multidegree refinement patterns, 2) extending the array-based half-facet (AHF) data structure [5] to support hierarchy generation and efficient mesh traversals, 3) developing efficient parallel communication strategies to resolve shared entities along processor boundaries after refinement, and 4) link with various high-order point projection strategies based high-order boundary reconstruction. We develop the capability under the parallel arraybased unstructured mesh framework "Mesh Oriented dAtaBase" a.k.a MOAB[21]). The developed mesh hierarchy generation supports 1D (edges), 2D (triangles, quadrilaterals), and 3D (tetrahedral, hexahedral) meshes and mixed-dimensional meshes. We present results to demonstrate the computational efficiency, memory requirements and effect on mesh quality due to templatebased UMR. We also demonstrate the effectiveness of the hierarchical meshes through a multigrid application.

A key aspect of the refinement algorithm is the positioning of the new vertices as entities are refined and we currently use a linear point projection scheme. However, using linear point projection scheme for the new vertices compromises the accuracy of the geometry and in turn that of the finite element solver. To address this issue, we make use of a recently added discrete geometry module in MOAB that provides high-order point projection schemes. The discrete geometry module provides high-order boundary reconstruction strategies that are based on weighted averaging of local polynomial fittings as described in $[10,11]$. We present convergence studies of the geometrical errors using different point projection schemes for various mesh hierarchies.

This work is an extension of the conference paper[17] and improves the original paper on two aspects. First, we introduce an alternative approach based on a combinatorial matching algorithm to resolve shared interface entities. Secondly, we include a high-order boundary reconstruction capability to project the new points after refinement using high-order approximations in- stead of linear projection so that geometrical errors introduced by curved boundaries can be minimized. In [18], a parallel hierarchical tetrahedral-octahedral subdivision refinement scheme is described. The proposed scheme was specific to tetrahedral meshes whereas our framework is applicable to a larger set of entity types. Also, the refinement template in [18] leads to a significant jump in the number of entities comparing to using lower-degrees of refinements where the mesh size increase is more graded. Finally, the parallel model follows a master-slave communication model whereas we follow a purely distributed model with mesh partitioning along with various asynchronous communication strategies to overlap communication with computation for latency hiding. Our template-based approach is further extended in [27] to support both non-conformal and conformal adaptive refinement while using an arraybased tree-like structure for storing the hierarchies. In $[3,15,26]$, various strategies for adaptive mesh refinement for both unstructured and structured meshes are provided. Our approach, though still in preliminary stages, differs significantly. It first of all allows storing the hierarchy information and subsequently support various inter and intra level mesh traversal queries are required by multi-level methods. Secondly, unstructured meshes and as a result complex geometries can be directly supported without posing any constraint on the geometry. We refer to [4] for an overview of the existing adaptive strategies as detailed comparision is beyond the scope of this work.

The remainder of the paper is organized as follows. Section 2 reviews some background knowledge and related mesh data structures. Section 3 describes the refinement templates, underlying mesh hierarchy storage and extended half-facet data structures. Section 4 describes the algorithms for the parallel communication and mesh hierarchy generation. Section 5 presents numerical results for computational efficiency, memory requirements, effect on mesh quality, convergence properties of different point projection schemes, and an example of multigrid solver. Section 6 concludes the paper with a discussion.

\section{Related Work and Background}

\subsection{Background}

Mesh data structures are fundamental to meshing algorithms and mesh-based numerical methods. The underlying data structure strongly influences the overall performance of the algorithms or simulations, since it 
is used to perform all the mesh-based combinatorial operations and as a result has been investigated since the inception of mesh generation and computational geometry. We review some terminology before describing our data structures and mesh frameworks. We say a mesh is a manifold or non-manifold if its geometric realization is a manifold or non-manifold, respectively. In a $d$-dimensional mesh, we refer to the $d$-dimensional entities as elements, and refer to the (d-1)-dimensional subentities as its facets. Each facet in a 2-D or 3-D mesh has an orientation with respect to the containing element. For example, each edge of a triangle has a direction, and all the edges form an oriented loop. Thus, it makes sense to call the facets half-facets. Each facet may have multiple incident elements, especially for non-manifold entities. We refer to all such half-facets as sibling halffacets. A mesh is said to be conformal if the pairwise intersection of any two entities is either another entity (lower-dimensional) or is empty. In this paper, we consider only conformal meshes, which may be manifold or non-manifold. In some engineering applications, especially in coupled or multi-component systems, the domain of interest may be composed of a union of topologically 1-D, 2-D, and 3-D objects, such as a mixture of cables, thin-shells, and solids. We refer to such a domain and its mesh as mixed-dimensional. We refer to a subset of the mesh corresponding to a 1-D, 2-D, and 3-D object in the domain as a sub-mesh.

\subsubsection{Array-Based Half-Facet (AHF) Data Structure}

There are a number of mesh data structures such as entity-based, boundary representations, corner table, radial-edge, winged, half- edge/face, incidence graphs, etc., that are used for mesh representation and queries. The two data structures that are relevant in our context are the half-edge and half-face data structures. The halfedge data structure is for $2 \mathrm{D}$ and surface meshes. It uses edge as the core object where the edge within each face is called a directed or half-edge. Typical implementations (e.g., CGAL [6, 12], OpenMesh [2] and Surface_Mesh [20]) store mappings from each half-edge to its opposite half-edge, its previous and next half-edge within its face, its vertices, its incident face, as well as the mapping from each vertex and each face to an incident half-edge. More compact representations, such as [1], can be obtained by storing only the mapping between opposite half-edge, optionally the mapping from each vertex to an incident half-edge, along with the element connectivity. The half-edge concept was generalized to half-faces (e.g.,[1] and [14]) for volume meshes where half-faces refer to the oriented faces within a cell. These basic half-edge and half-face data structures are simple and are restricted to oriented, manifold meshes (with or without boundary) in 2-D and 3-D, respectively.

In [5], an efficient, compact and general arraybased half-facet (AHF) mesh data structure with support for mixed-dimensional meshes, which may be nonmanifold and/or non-oriented was proposed. The core object of AHF is half-facet as defined previously and is represented as an implicit entity. The concept of sibling half-facets unifies the half-vertex, half-edge, and half-face data structures for 1-D, 2-D, and 3-D meshes, which may be manifold or non-manifold with boundary. The AHF data structure consists of two key maps:

- sibling half-facets (sibhfs): The mapping between the sibling half-facets using a cyclic linked list.

- vertex to half-facet $(v 2 h f)$ : The map of each vertex to its incident half-facet. This map provides an anchor for each vertex to its locality in the mesh.

An example of the AHF maps for a non-manifold mesh is illustrated in Figure 1a. For $d \geq 2$, AHF provides a compact representation, since the intermediate dimensional entities are not stored but referenced implicitly. This data model stores the above two maps for each dimension in a modular and self-contained manner and hence supports mixed-dimensional meshes, which may be composed of sub-meshes of 1-D, 2-D, and 3-D. Figure $1 \mathrm{~b}$ shows a diagram of a typical half-facet data structure, where the half-vertices and half-edges are only required for explicit edges and faces in the mesh, respectively. In addition, this data model can also be used for meshes with high-order elements (such as six-node triangles or 10-node tetrahedra), where the mid-edge, mid-face or mid-cell nodes do not affect the definition and identification of the half-facets. We use the AHF as the underlying mesh data structure for developing the refinement algorithms.

\subsubsection{Mesh Oriented datABase (MOAB)}

To be of any practical use to numerical simulation workflows, the mesh framework needs to support a wide range of functionalities such as efficient local mesh traversals for matrix assemblies, boundary extraction for boundary conditions, efficiently support adjacency and connectivity queries, representation of mesh data, etc., in a parallel setting. Over the past years, a number of such frameworks have been developed e.g., FMDB [19], MSTK [8], libMesh [13], etc, with various mesh representations addressing application specific needs. In such implementations, the entities are represented as "objects" explicitly, and pointers (or handles) are used to refer to these explicit objects. In our work, we choose 


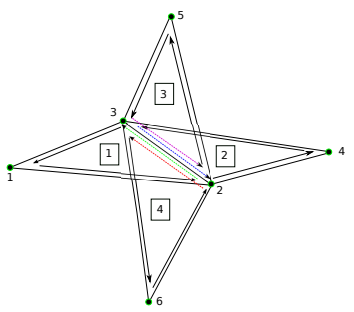

\begin{tabular}{|c|c|c|c|}
\hline element & \multicolumn{3}{|c|}{ connectivity } \\
\hline 1 & 1 & 2 & 3 \\
\hline 2 & 2 & 4 & 3 \\
\hline 3 & 2 & 5 & 3 \\
\hline 4 & 2 & 3 & 6 \\
\hline
\end{tabular}

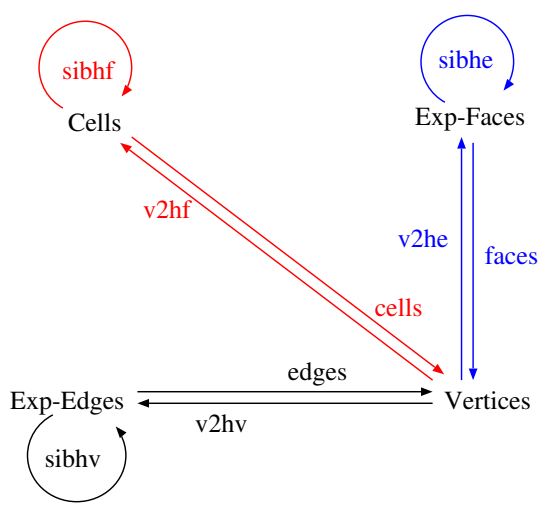

(a) AHF maps for a non-manifold mesh.

(b) AHF maps for mixed-dimensional meshes.

Figure 1: (a) An example of the AHF maps stored for a non-manifold triangular mesh. (b) Typical AHF for mixed-dimensional meshes is composed of half-vertex (black, for explicit edges only), half-edge (blue, for explicit faces only), and half-face (red) data structures.

to use array-based, pointer-free implementations for a number of reasons. First, using arrays can lead to faster memory access, fewer cache misses and hence better efficiency. Secondly, in an array-based implementation, we can treat intermediate dimensional entities (such as half-facets) as implicit entities, and reference them without forming explicit objects. This can lead to significant savings in storage, especially on computers with 64-bit pointers. In addition, array-based implementations also offer better interoperability across application codes, different programming languages, and different hardware platforms (such as between GPUs and CPUs).

One such array-based parallel mesh framework is the Mesh Oriented datABase a.k.a MOAB [21] which is a mesh data representation designed to support a range of mesh related operations, such as memory efficient mesh representation, mesh querying and representation of application specific data. Internally it uses an array-based storage for fine-grained data, which in many cases provides more efficient access, especially for large contiguous portions of a mesh and its associated data. The AHF data structure was implemented in MOAB to support query-intensive algorithms, especially those involving non-vertex/higher-dimensional adjacency queries and was found to be extremely efficient (in some cases over two orders of magnitude improvement $[5,16]$ ) over the existing data structures. MOAB is also a parallel framework and supports spatial domain-decomposed view of a parallel mesh where each subdomain is assigned to a processor, lower-dimensional entities on interfaces between subdomains are shared between processors, and ghost entities can be exchanged with neighboring processors. We develop our hierarchical mesh generation algorithms on top of MOAB's parallel framework. In the next section, we describe the three key components of the proposed hierarchical mesh generation algorithm.

\section{Multi-Degree, Multi-dimensional and Multi- Level Hierarchical Meshes}

The uniform refinement based mesh hierarchy generation has three key design components. First, entity type and degree-specific refinement templates are defined that are used to subdivide an entity into its children. The templates are also used to update the underlying data structures for all the new children. The second key design component is the use of array-based halffacet data structures for efficient mesh traversal during refinement. Finally, the newly created meshes are stored in level-wise contiguous array to provide memory compactness.

\subsection{Multi-degree Refinement Templates}

The standard refinement strategy divides each $d$ dimensional entity to $1 \rightarrow 2^{d}$ subentites. However, a desired mesh resolution can be reached much faster if a higher degree of refinement is used. In such cases, the length of the mesh hierarchies is also small, which might be preferable from some multigrid methods such as GMGs. Another motivation to use high degree refinement patterns is that it allows straightforward extension 
to high-order entities and thus support $h p$-refinements. By multi-degree refinement we mean a refinement pattern following vertex positions analogous to high-order (degree $p$ where $p \geq 2$ ) Lagrangian elements. Thus, for a $d$ dimensional entity, a degree $p$ refinement divides the entity to $1 \rightarrow p^{d}$ subentites. We support prime number degrees as any higher degree refinements can be obtained by applying a sequence of such prime degree refinements. Table 1 lists the currently supported degrees for each dimension. We note that in uniform refinement the same modification operation of dividing an entity into a specific number of entities is applied to each entity of the mesh. Thus defining static templates w.r.t a reference entity that contains certain entity type and degree specific information could aid the refinement process. The template mainly stores the following information for each entity type and supported degree of refinement :

1. Numbering convention of new vertices: The new vertices are assigned local indices through which they are uniquely identified within a reference entity.

2. Connectivity of new child entities: The connectivity of the new entities using the local indices of the new vertices. Each such child is also uniquely identifiable by a local id with respect to the reference entity.

3. Half-facet maps for new child entities: The local sibling half-facet (refTemplate $\rightarrow$ sibhfs) and vertex to incident half-facet (refTemplate $\rightarrow \mathbf{v} \mathbf{2 h f}$ ) maps are stored. Note that these maps use the local child entity id's and follow the ordering convention defined in the template.

Figure 2 shows the templates for triangle and quadrilateral reference entities for degrees 2 and 3. The numbering convention of the reference entity follows the MOAB canonical numbering. Apart from these three pieces of data, the template also stores some other auxiliary information to aid tracking of new vertices introduced on the entity boundary to avoid vertex duplication during refinement. An example of a degree 3 refinement template for a reference triangle is illustrated in Tabl 2.

Except for tetrahedron entity, the templates are static for all other entities (triangles, quads, hexes) in the sense that they do not need to refer to the physical entity in order to refine the parent entity. The refinement schemes for a tetrahedron involves division into smaller congruent tetrahedra and octahedrons which are subsequently divided into four tetrahedra. Figure 3 shows how refining a tetrahedron leads to congruent smaller tetrahe-
Table 1: The degrees of refinement currently supported and the corresponding number of children.

\begin{tabular}{|c|c|c|}
\hline Dimension & Degrees & \#Children \\
\hline \hline 1 & $2,3,5$ & $2,3,5$ \\
\hline 2 & $2,3,5$ & $4,9,25$ \\
\hline 3 & 2,3 & 8,27 \\
\hline
\end{tabular}

Table 2: An example for the degree 3 template of a triangle reference entity. Children entity connectivity and the template $v 2 h f$ and sibhfs maps.

\begin{tabular}{|c|c|}
\hline \multicolumn{2}{|c|}{ Child Entity Connectivity } \\
\hline 1 & $\langle 0,3,8\rangle$ \\
\hline 2 & $\langle 3,9,8\rangle$ \\
\hline 3 & $\langle 3,4,9\rangle$ \\
\hline 4 & $\langle 4,5,9\rangle$ \\
\hline 5 & $\langle 4,1,5\rangle$ \\
\hline 6 & $\langle 8,9,7\rangle$ \\
\hline 7 & $\langle 9,6,7\rangle$ \\
\hline 8 & $\langle 9,5,6\rangle$ \\
\hline 9 & $\langle 7,6,2\rangle$ \\
\hline
\end{tabular}

\begin{tabular}{|l|l|l|}
\hline \multicolumn{3}{|c|}{ sibhfs } \\
\hline$\langle 0,0\rangle$ & $\langle 2,2\rangle$ & $\langle 0,0\rangle$ \\
\hline$\langle 3,2\rangle$ & $\langle 6,0\rangle$ & $\langle 1,1\rangle$ \\
\hline$\langle 0,0\rangle$ & $\langle 4,2\rangle$ & $\langle 2,1\rangle$ \\
\hline$\langle 5,2\rangle$ & $\langle 8,0\rangle$ & $\langle 3,1\rangle$ \\
\hline$\langle 0,0\rangle$ & $\langle 0,0\rangle$ & $\langle 4,0\rangle$ \\
\hline$\langle 2,1\rangle$ & $\langle 7,2\rangle$ & $\langle 0,0\rangle$ \\
\hline$\langle 8,2\rangle$ & $\langle 9,0\rangle$ & $\langle 6,1\rangle$ \\
\hline$\langle 4,1\rangle$ & $\langle 0,0\rangle$ & $\langle 7,0\rangle$ \\
\hline$\langle 7,1\rangle$ & $\langle 0,0\rangle$ & $\langle 0,0\rangle$ \\
\hline
\end{tabular}

\begin{tabular}{|c|c|}
\hline $\mathrm{v}$ & $v 2 h f$ \\
\hline 0 & $\langle 1,0\rangle$ \\
\hline 1 & $\langle 5,1\rangle$ \\
\hline 2 & $\langle 9,2\rangle$ \\
\hline 3 & $\langle 3,0\rangle$ \\
\hline 4 & $\langle 5,0\rangle$ \\
\hline 5 & $\langle 8,1\rangle$ \\
\hline 6 & $\langle 9,1\rangle$ \\
\hline 7 & $\langle 6,2\rangle$ \\
\hline 8 & $\langle 1,2\rangle$ \\
\hline 9 & $\langle 8,0\rangle$ \\
\hline
\end{tabular}

dra and octahedra. The right side of the figure shows the three possible diagonal choices for each such octahedron to be divided into four tetrahedra. To deliver a good mesh quality, each such octahedra is divided into sub-tetrahedra by connecting the shortest diagonal [25]. Thus, for higher-degree refinements, each octahedron has to find the shortest diagonal using the physical entity. However, we note that though the smaller tetrahedra obtained by tessellating an octahedron are not congruent to the parent tetrahedron, all the octahedra are congruent to each other. Therefore, the shortest diagonal is unique and hence each octahedron would be divided using the same pattern. We use this fact to define three static templates for each degree. During refinement, each tetrahedron makes a choice of the appropriate template based on the smallest diagonal of the physical tetrahedron. Currently, the template generation is not automatic. Effort is being made to make it automatic so that higher-degrees, such as 7,11 , etc., can also be supported.

\subsection{Multi-dimensional Mesh Data Structures}

During refinement, one of the key tasks is to avoid introducing duplicate vertices for shared boundaries between entities. This requires frequent calls to adjacency routines to get entities connected through entity bound- 


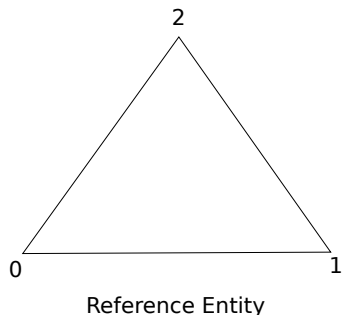

Reference Entity

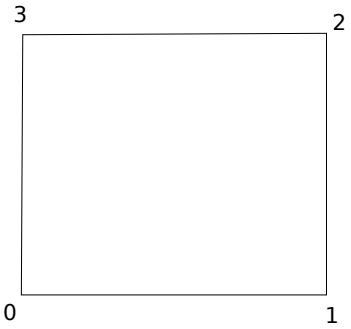

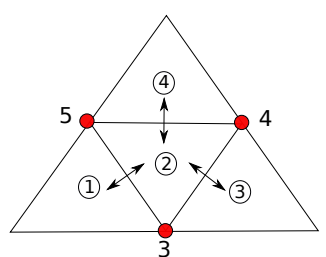

Degree 2

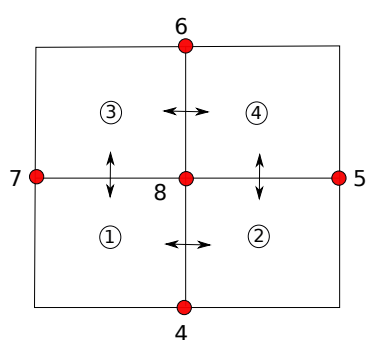

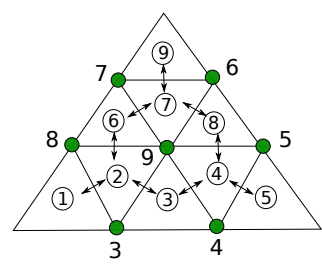

Degree 3

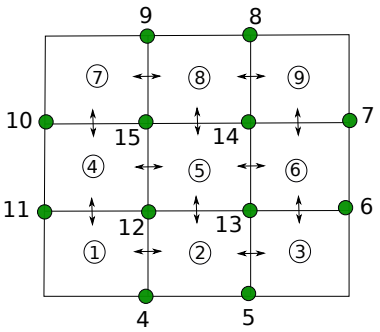

Figure 2: The refinement templates defined for degree 2 and 3 refinements over a reference triangle and quadrilateral entity. The local vertex order of the reference entities follow MOAB conventions [22]. The new vertices to be introduced during refinement are assigned local ids w.r.t. to the reference. Similarly, the children entities are assigned local order. The AHF maps are created for the refined entities in terms of their local ids.
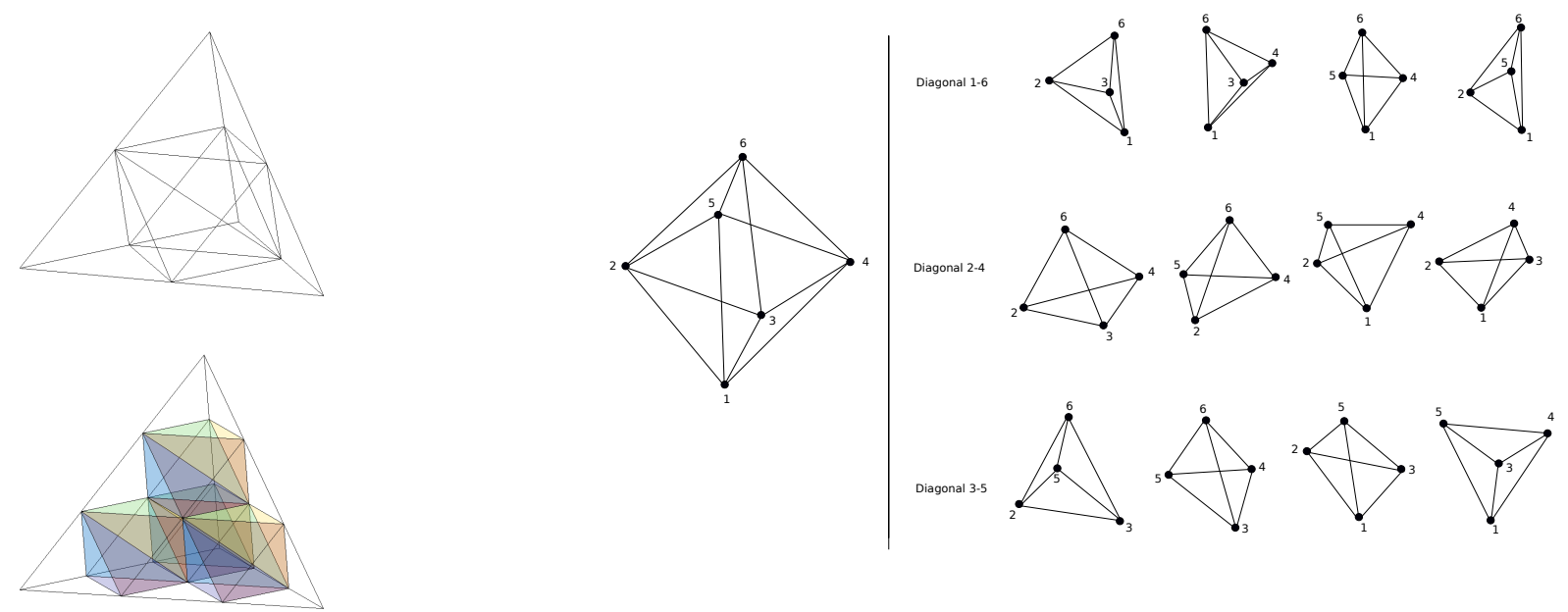

Figure 3: Left: A tetrahedron is divided into congruent smaller tetrahedra and octahedrons. Middle: A reference octahedron. Right: Each octahedron can be further subdivided into four tetrahedra in three possible ways depending on which diagonal is chosen. 
aries. Since AHF stores the half-facet maps between entities, these types of queries are the most efficient ones it can support. As a result, it is natural to use AHF as the underlying data structure. As each level of the mesh hierarchy is generated by refinement of the previous level, it is necessary to update the appropriate AHF maps for the new level so that the new mesh can be queried later. The process of updating the AHF maps for the new level is aided both by the refinement templates (defined in the previous subsection) and also by the fact that the topology of the domain does not change during refinement. Thus, children of manifold entities remain manifold for all levels, and no new non-manifold entities are introduced. The AHF maps for a refined mesh are updated in two stages:

1. Update maps for children of an entity: After an entity in the coarse mesh is refined, the sibling half-facet (sibhfs) and vertex-to-incident half-facet (v2hf) maps are updated only for the child entities of the working entity by using the refTtemplate $\rightarrow$ sibhfs and refTtemplate $\rightarrow$ v $2 \mathrm{hf}$ maps defined in the refinement templates.

2. Update maps between children of parent siblings: After all the entities of the coarse mesh have been refined, the global half-facet maps are now updated to connect the child entities incident on boundaries of the parent entities.

Figure 4 illustrates the above two steps during refinement of a triangle mesh with two entities. Algorithms 1 and 2 outline the two-step procedure for updating the AHF maps for a mesh level. For mixed-dimensional meshes, the maps for each lower dimensional submesh are updated in a similar manner.

\subsection{Multi-level Mesh Storage}

An array-based mesh storage leads to increased efficiency, but it requires careful consideration to maintain it for operations involving a change in the contiguity of the memory space. By virtue of the uniform refinement of the mesh, it is possible to estimate the total number of entities that will be created for a given degree of refinement. Table 3 shows the estimates for new entities created after a single level of refinement for a 3D mesh with embedded curves and surfaces. Once the storage requirement for a new level is estimated, the memory is allocated in contiguous blocks. During refinement, as each entity is subdivided, the new entities are stored according to the local order specified in the refinement template. Thus, children of the first entity in previous
Table 3: Estimates for new entities that will be created after a refinement of degree $\mathrm{p}$. These estimates are for a 3D mesh with explicit curves and surfaces. Here $|\cdot|$ denotes the number of entities in a particular entity set. $E^{p}$ and $F^{p}$ are the total number of edges and faces in the $3 \mathrm{D}$ mesh at the previous level, respectively.

\begin{tabular}{|c|c|c|}
\hline & \#Entities at previous level & \#Entities after refinement of degree $p$ \\
\hline \hline Vertices & $\left|V^{p}\right|$ & $|V|=\left|V^{p}\right|+n v e *\left|E^{p}\right|+n v_{f} *\left|F^{p}\right|+n v_{C} *|C|$ \\
\hline Explicit Edges & $\left|E_{\text {exp }}^{p}\right|$ & $\left|E_{\text {exp }}\right|=p *\left|E_{\text {exp }}^{p}\right|$ \\
\hline Explicit Faces & $\left|F_{\text {exp }}^{p}\right|$ & $\left|F_{\text {exp }}\right|=p^{2} *\left|F_{\text {exp }}^{p}\right|$ \\
\hline Cells & $|C|$ & $|C|=p^{3} *\left|C^{p}\right|$ \\
\hline
\end{tabular}

level are stored first, then the children of the second and so on. This data layout supports straightforward index based inter-level (i.e., parent-to-child or child-to-parent) queries, which are vital to multi-level methods. Figure 5 illustrates this memory layout. To provide mesh independence at each level, we duplicate vertices from the previous level to create the new mesh along with new vertices. As a result, the mesh hierarchy generation would return a sequence of meshes that are independent of each other while providing inter- and intra-level mesh access. If necessary, the vertices at a specific level can be renumbered to maintain small bandwidths for the assembled matrices.

\section{Hierarchical Mesh Generation in Parallel}

Generating mesh hierarchies using uniform refinement in parallel may seem to be a relatively straightforward process, since each processor refines its local mesh. If the initial mesh distribution is balanced, then uniform refinement would not introduce any additional imbalance. Thus, there is no need to move mesh entities between processors. However, generation of each level of the hierarchy also introduces new entities on the shared interface between processors. Unless these new entities are resolved, the generated hierarchies are only useful for local operations. More complicated algorithms requiring shared information such as exchanging ghost-layers, solver setups that rely on knowing owned/ghosted entities for DoF distribution would break down. In this section, we discuss two parallel communication algorithms that resolve such new entities on the shared interface between processors. We also describe the refinement algorithm to generate multi-degree, multi-dimensional hierarchies of unstructured meshes in parallel. 


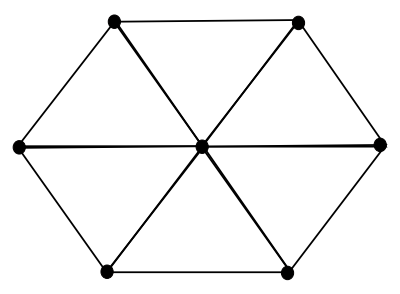

Degree-3 Refinement

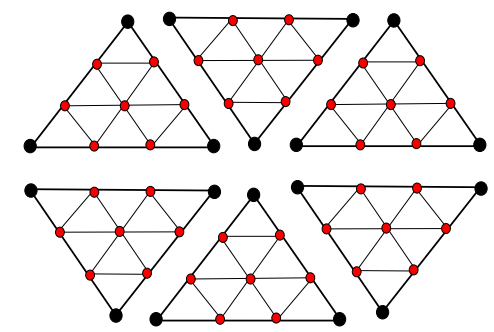

AHF maps for child entities

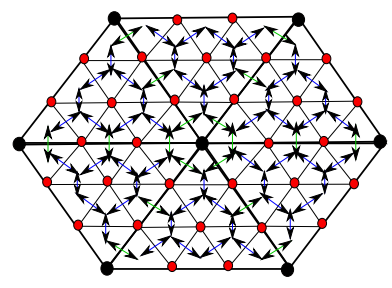

Global Update:
AHF maps for children on shared facet of parents
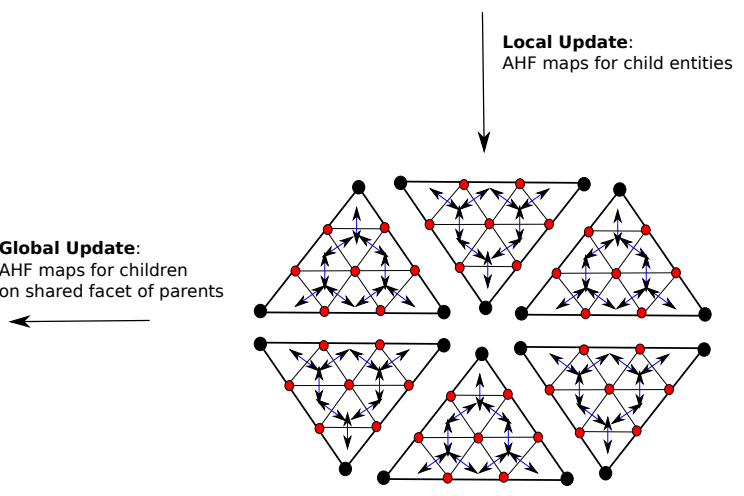

Figure 4: Updating the AHF maps for the refined mesh takes place in two stages. First, maps for the children of each triangle are updated. After, both the triangles have been refined, the maps are now updated to connect the children entities sharing a parent facet.

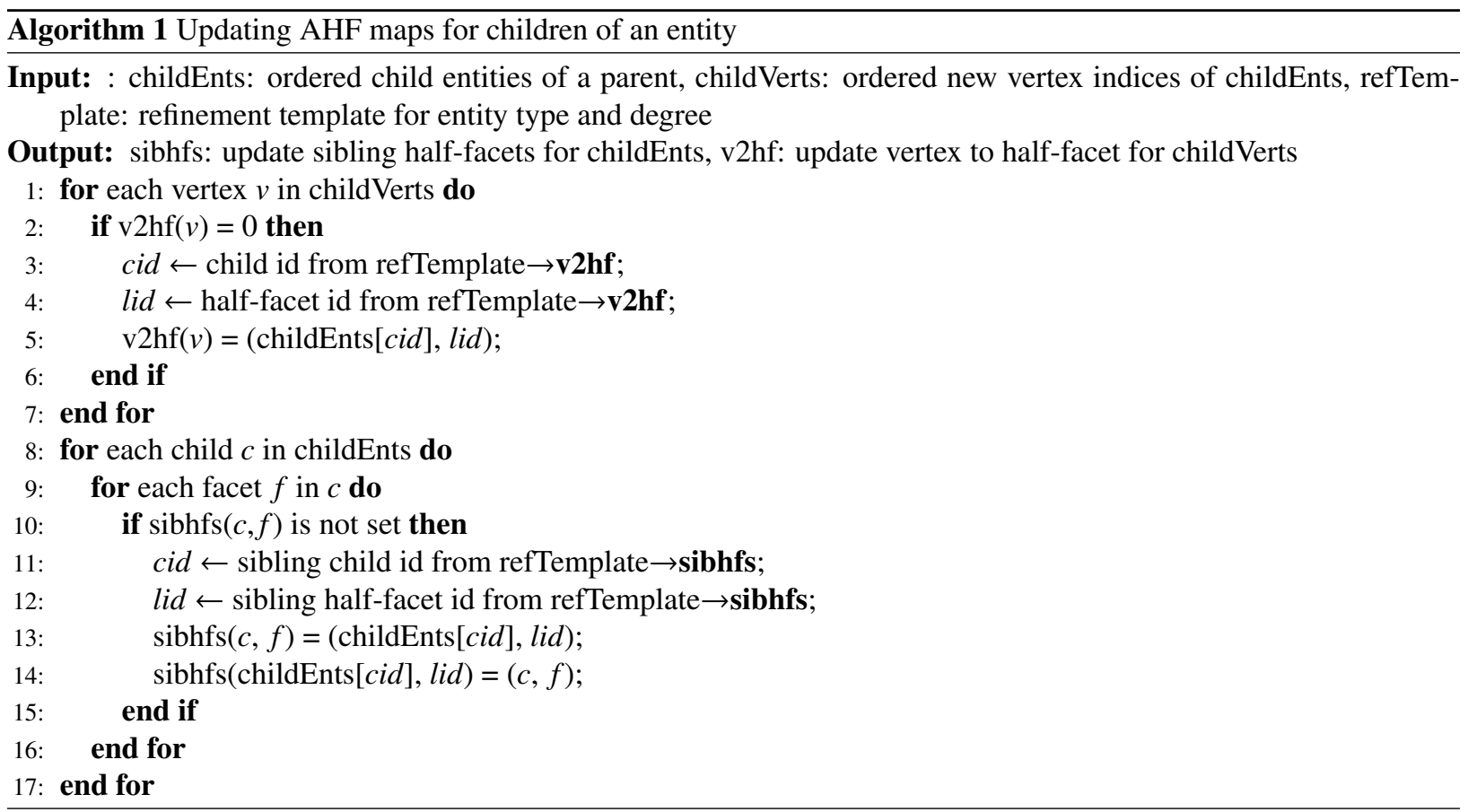



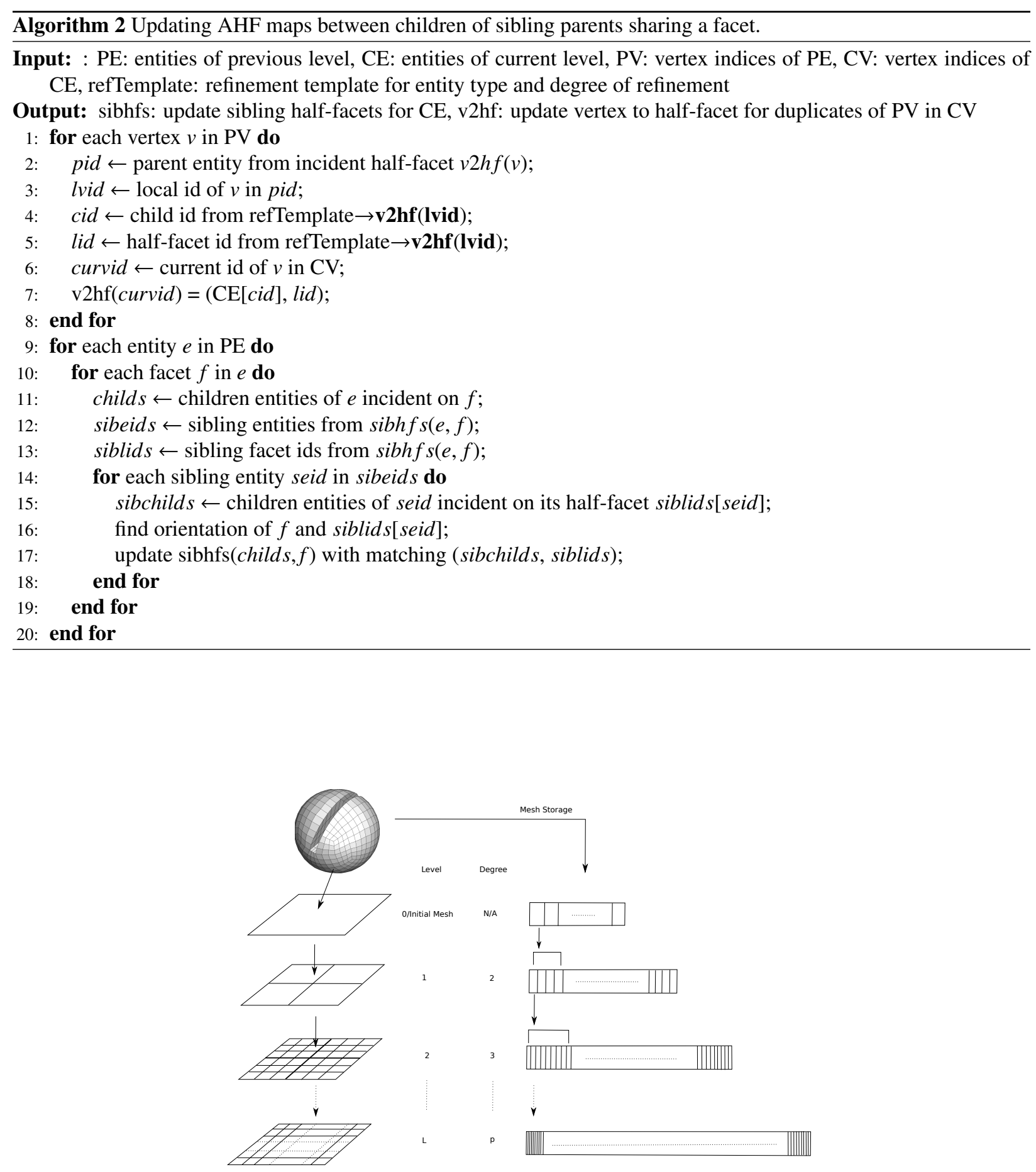

Figure 5: The data layout of meshes at each level. For example, starting with the first quad of the sphere mesh, after each level of refinement, the children are stored in a pre-decided order as defined in the refinement templates. This local order uniquely identifies each child of the parent. 


\subsection{Parallel Communication to Resolve Shared Enti- ties}

The MOAB library implemented with array-based data-structures have been designed to be scalable in memory layout and access. It supports a distributed MPI-based communication model to target applications based on similar approach. There are several optimized one-sided communication algorithms that make use of aggregation strategies to minimize total data transferred between processors. Using this parallel framework, the mesh hierarchy generation can be performed in a series of optimal steps. Once each processor loads a part of the distributed coarse mesh, local refinement for all the entities can be performed. However, the new entities on the shared interface created by the refinement have to be resolved, so that other communication algorithms requiring shared mesh or data can take place. This is necessary to avoid writing out duplicate shared entities as well as for supporting in-memory operator discretization over the refined levels. We discuss the following two approaches to communicate and resolve these new shared entities:

- The first approach uses a parallel merge algorithm using geometric proximity of the mesh vertices to resolve first the shared vertices and subsequently the subentities.

- The second approach uses a combinatorial matching algorithm using the coarse mesh to match local handles of new entities with their remote handles.

We first provide a brief description of the key parallel information that is stored in MOAB for distributed meshes. In MOAB, the parallel information for a distributed mesh consists of the following:

- PSTATUS: This is a flag classifying the type of sharing information for an entity with five possible classifications: owned, interface, ghost, shared, and multishared.

- SHARED: In the case an entity is shared with only one other process, the entity is classified as shared, and its remote handle and the remote processor rank is stored.

- MULTISHARED: In the case an entity is shared with more than one other process, the entity is classified as multishared, and all of its remote handles in remote processors are stored including the current processor and local handle.
These three fields are stored in special tags (a datatype in MOAB for storing data over mesh entities) for entities belonging to each partition and can be created during the mesh loading step with explicit calls to resolution of shared interface through the mesh loading function interface. The parallel infrastructure in MOAB provides native algorithms to resolve shared interfaces using GLOBAL ID's as well as geometric proximity along with an exchanging ghost layers and other parallel capabilities that are described in detail in [24]. During parallel refinement we do not refine any entities belonging to the ghost layers of the initial mesh.

Now, any algorithm for resolution of entities on the shared interface for the refined meshes has to create the above three kind of information correctly for the new entities. Since the refinement is based on pre-defined templates, the co-ordinates for the new vertices and entities on the shared interface between processors will be the same. We utilize this information to design a communication algorithm to resolve the newly created entities in preparation for synchronization of ghost layers and exchange of meta-data (MOAB tags[21]).

\section{Merge based resolve interface algorithm}

In our first approach, we use the parallel merging algorithm described in [9, 24] for joining meshes distributed on different processors to resolve the new vertices of the refinement levels. In this method, vertices on the shared interfaces are matched based on their geometric position. The algorithm proceeds by first partitioning equally the global bounding box, with each processor responsible for a distinct geometric region (plus a small epsilon layer whose thickness is twice the distance tolerance of the merge). In the first round of communication, each vertex from the skin of the local mesh is sent in a tuple containing its handle, its coordinates and its processor to the responsible processor. At the responsible processor, shared vertices are identified based on their coordinates, and the processor that will own each shared vertex is determined. In a second round of communication, each shared vertex information is sent back to originating processors, with the information about the other processors that share it, and its corresponding handles on those processors. These two rounds of communication use a sparse, gather-scatter communication algorithm (crystal router [7]). The non-vertex entities on the skin are resolved by using their connectivities, in a next step, using another round of asynchronous communication, and using the communication pattern determined from vertex sharing information. One disadvantage of the above merge algorithm is that it does not use the communication pattern that is already available 
from the coarsest mesh, leading to use of global communications instead of one-sided communication.

\section{Combinatorial matching based resolve interface algo- rithm}

To overcome the shortcoming of multiple rounds of global communications in the merge-based approach, we design an optimized algorithm for the resolution of the shared entities by taking into account the communication pattern of the coarsest mesh. Now, we note that the local and remote representation of any shared interface entity may differ as shown in Figure 6. However, the remote interface entity can be oriented to match the local representation via a combination of its connectivity and subsequently, its local and remote child entities. For example, in 2D (Figure 7), the local child edges can be matched with the remote child edges depending on the orientation difference between the coarsest local edge and its remote edge. This observation along with the fact that the communication pattern for each processor does not change during and after hierarchy generation forms the basis of the optimized resolve shared entities algorithm.

The combinatorial algorithm starts by gathering a list of shared processors with the current processor. For each shared processor, a list of coarsest level entities are collected along with their children entities and their connectivities. This information is then send to the sharing processor while ensuring that the local handles of the coarsest entities are replaced with their remote handles on the receiving processor. On the receiving processor, the connectivity of the coarsest entities now follows the order on the sending processor leading to detection of the combination difference between the local and remote representations. After this single round of one-sided communication, each process contains all the remote handles of new entities from its sharing processors. The algorithm then proceeds to decipher the type and match the local and remote handles of the new entities and finally updating the parallel information as described above. The outline of the optimized algorithm is given in Algorithms 3,4 and 5.

\subsection{Refinement Algorithm}

Figure 8 shows the flowchart of the refinement algorithm to generate a hierarchy of unstructured meshes in parallel. We have also developed a tool to expose this
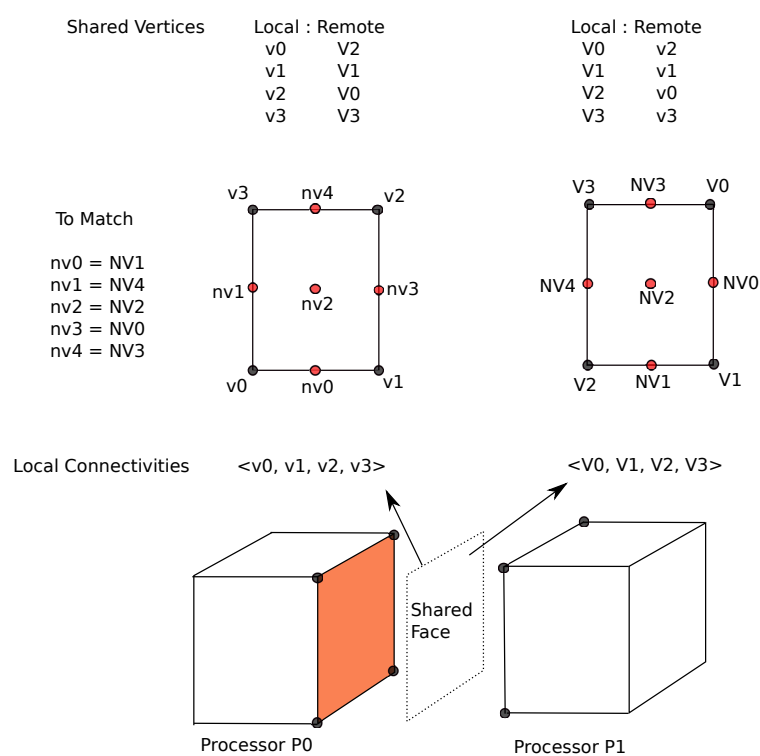

Figure 6: A shared face between two processors. After one level of refinement the new vertices and faces have to be matched.

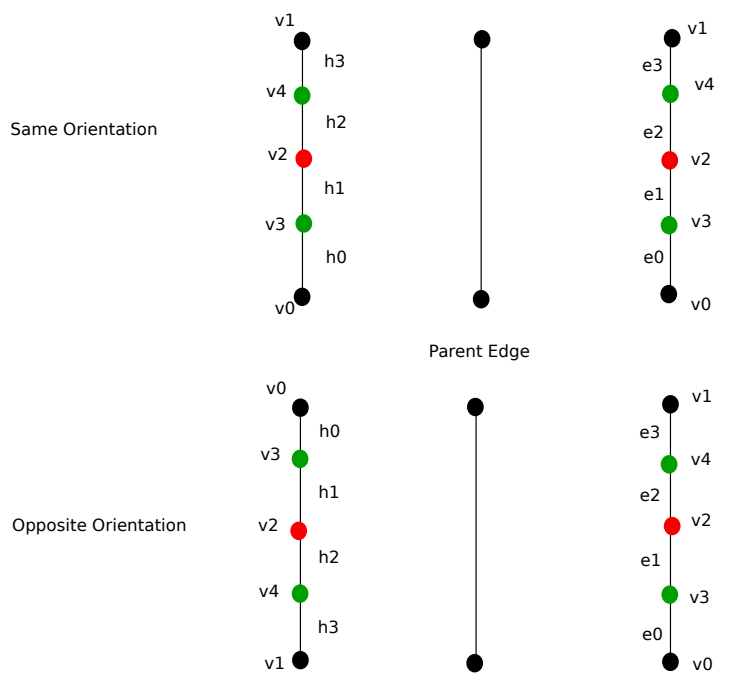

Figure 7: For a shared edge, there can be two options: either the remote edge has the same orientation or opposite orientation. Depending on the orientation, the new vertices and child edges can be appropriately matched. 

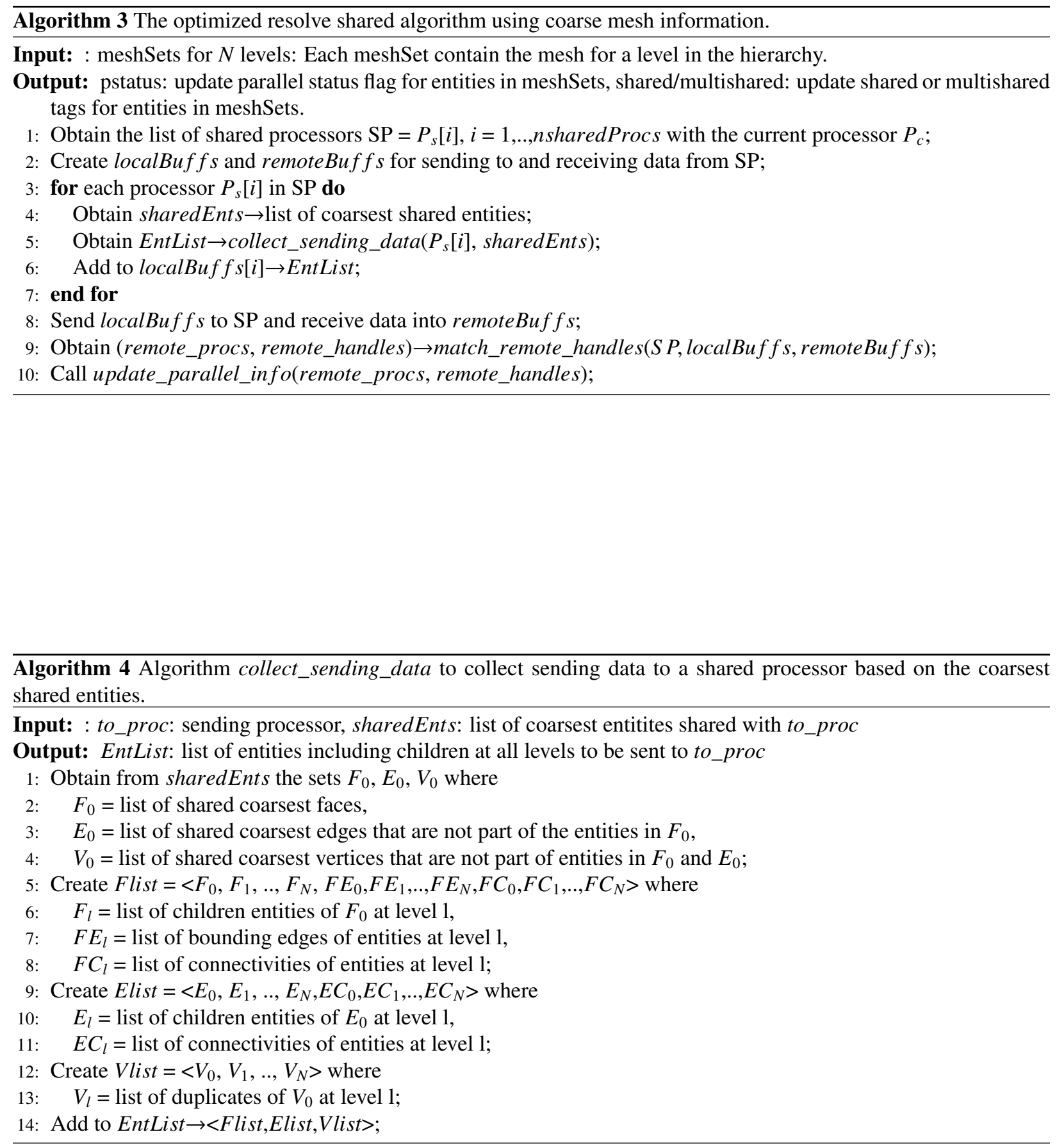


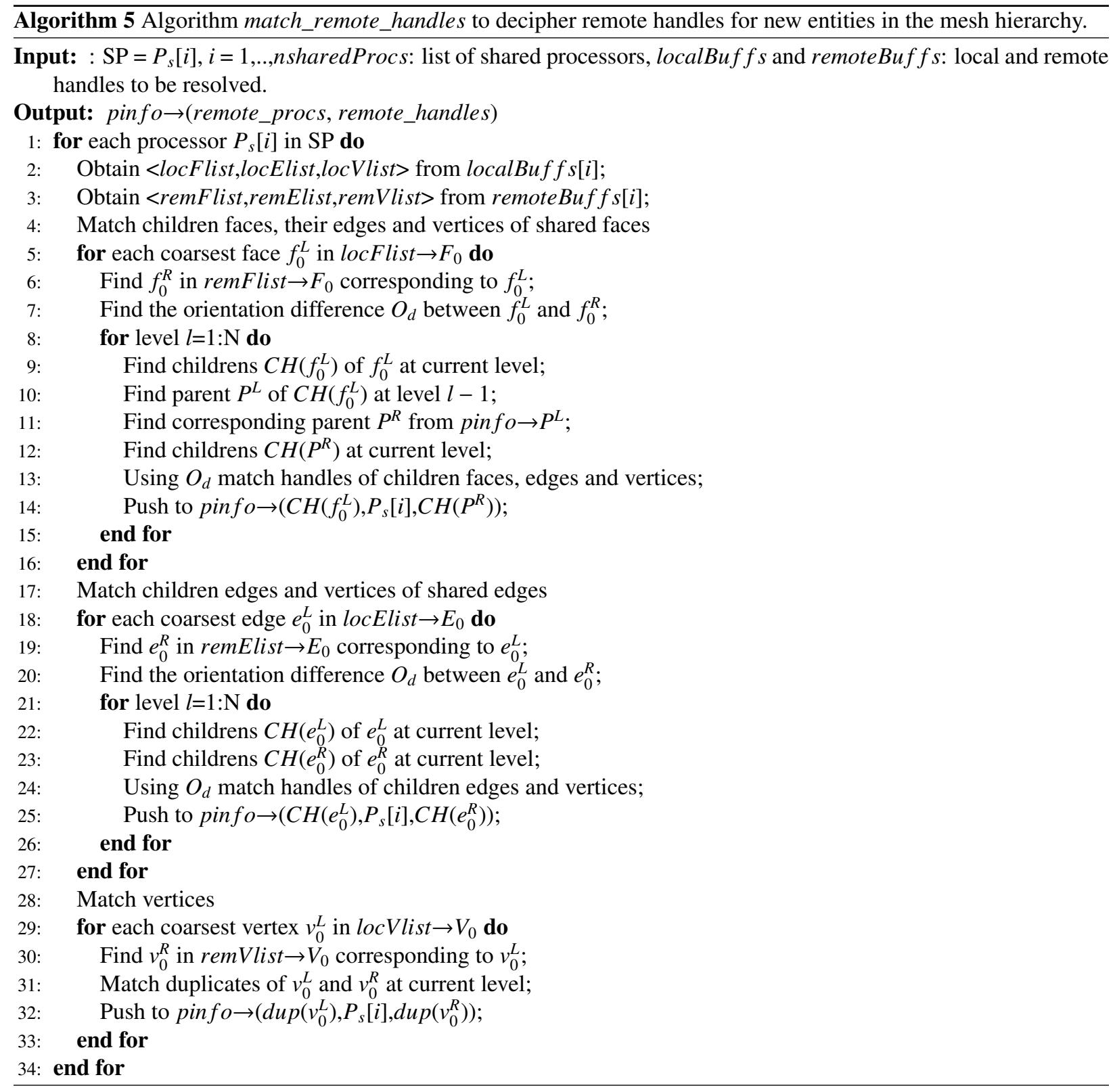


functionality in MOAB that can load a mesh, generate the hierarchies in parallel for a given number of levels and a sequence of degree of refinements, and then write back to a HDF5 file for consumption of the mesh hierarchy in PDE solvers.

\section{Numerical Results}

We present numerical results to demonstrate the effectiveness of uniform mesh refinement, in terms of computational efficiency of the parallel framework and its effect on mesh quality. We also demonstrate the application of the developed capability to study convergence properties of different point projection schemes for various mesh hierarchies as well as its application to multigrid method.

\subsection{Mesh Quality Under Uniform Mesh Refinement}

We first study the effect of uniform mesh refinement on the mesh quality. Since uniform refinement of tetrahedral meshes do not produce congruent sub-tetrehedra, we use it as our test case. An initial coarse tetrahedral sphere mesh with 23636 tets and 4793 vertices (shown in Fig. 9) was refined using three strategies all starting with the coarse mesh:

1. one degree-2 refinement,

2. one degree- 3 refinement, and

3. first a degree- 3 refinement followed by a degree- 2 refinement.

We use scaled Jacobian as the mesh quality measure. Figure 10 shows the distribution of the mesh quality measure for the meshes obtained using the three strategies along with the initial mesh. In all the three cases, the overall mesh quality improved with the shortestdiagonal approach [25] , and degree-3 refinement delivers similar and even slightly better quality improvement because there are more intermediate octahedra in degree-3 refinement.

\subsection{Computational Efficiency}

We now report the computational efficiency results of the mesh hierarchy generation algorithm. We begin with an estimation of the serial computational cost. Let $N_{0}$ be the number of entities in the initial coarse mesh of dimension $D$ on which a sequence of length $L$ of refinements with degrees $r_{i}, i=1, \cdots, L$ are applied. Thus, the total number of entities for any level $l$ where

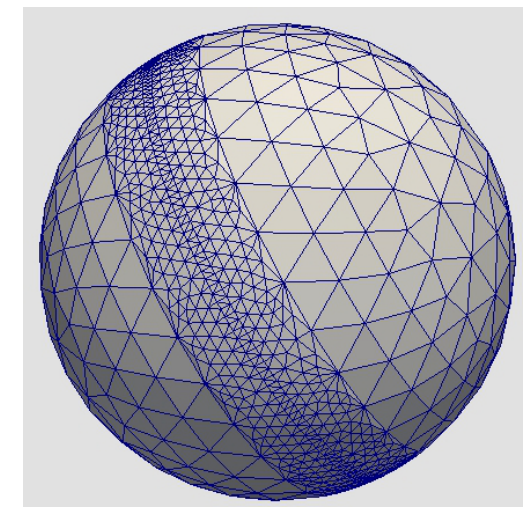

Figure 9: A tetrahedral mesh.

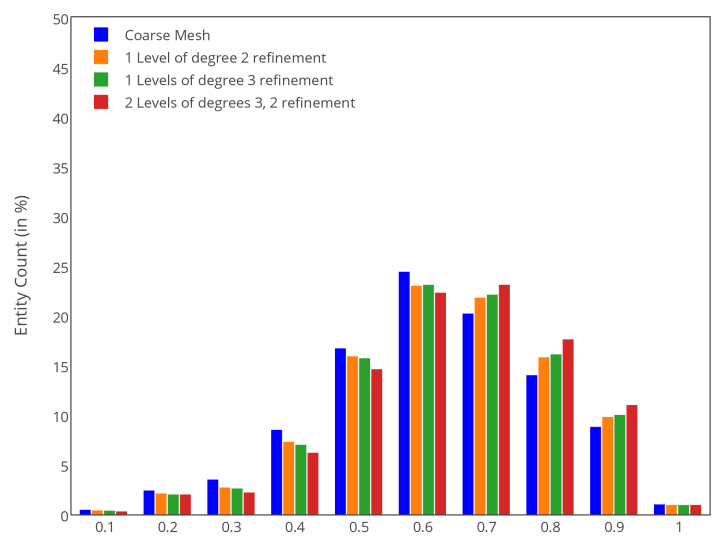

Mesh Quality (Scaled Jacobian)

Figure 10: Distribution of mesh quality for an initial coarse mesh and various degree of refinements. 


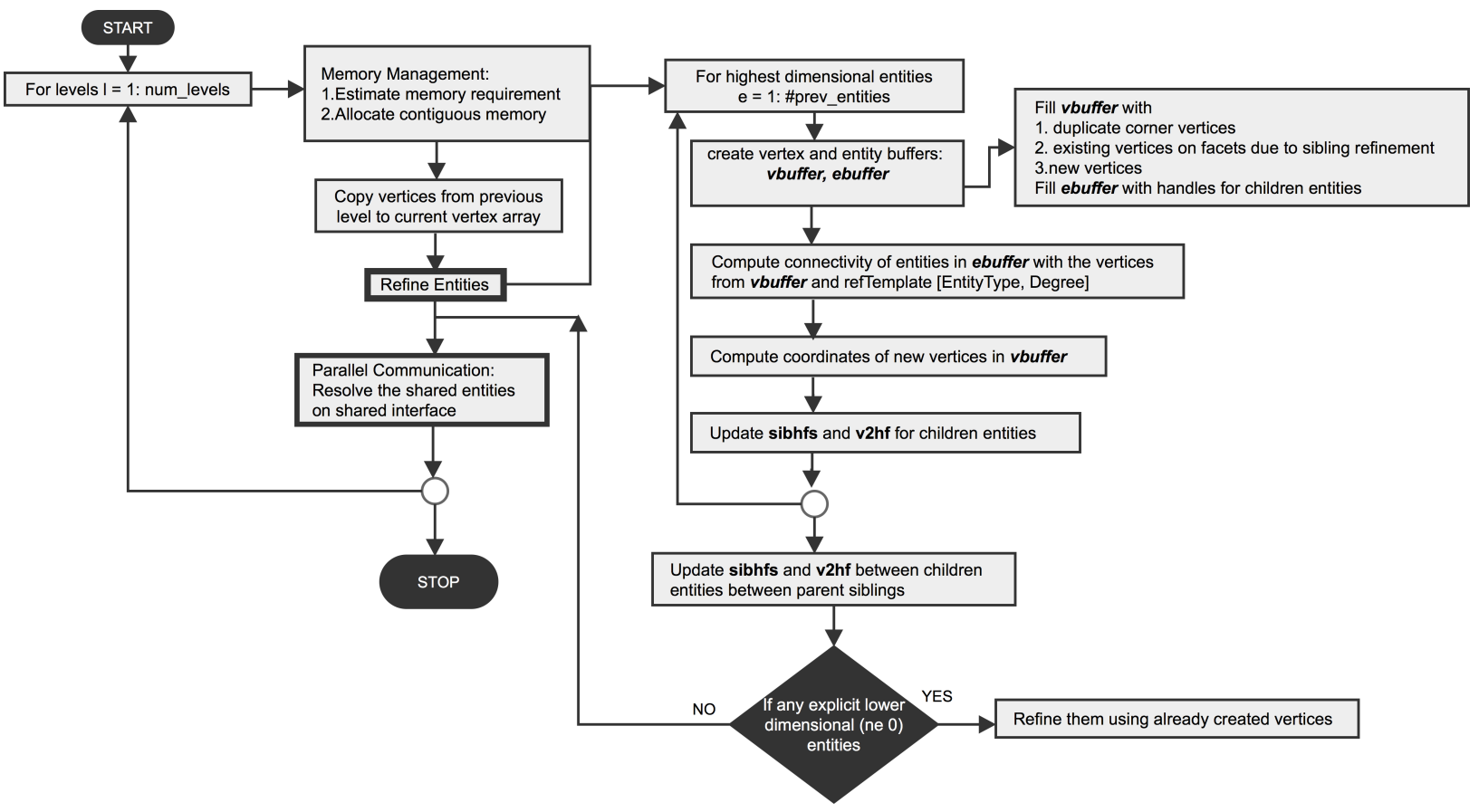

Figure 8: The flowchart for generation of mesh hierarchy with length num_levels.

$1 \leq l \leq L$ is given by $N_{l}=N_{0} r_{1}^{D} r_{2}^{D} \cdots r_{l}^{D}$. Assuming $r_{1}=r_{2}=\cdots=r_{L}=r$, we get $N_{l}=N_{0} r^{l D}$. Clearly, the time taken to refine a single level is $O\left(N_{l-1}+N_{l}\right)$ where $N_{l-1}$ are the number of entities in the previous level and the $N_{l}$ contribution comes from updating the mesh data structures for the new level. Without loss of generality, we can then write $t(N)=C . N$ where $\mathrm{C}$ is the constant and $N$ is the number of entities. We also try to provide an estimate for this constant. Let $t_{1}$ be the time taken to refine $N_{0}$ entities with degree $r$. Clearly, it is reasonable to expect that the refining the next level i.e., level 2 should take approximately $t_{2}=r^{D} t_{1} \Longrightarrow r^{D} t_{1}=C N_{0} r^{2 D} \Longrightarrow C=t_{1} /\left(N_{0} r^{D}\right)$ . We observe this linear behaviour in our numerical experiments.

In Figure 11, the serial run times for generating hierarchies using different supported degree of refinements on representative meshes for two and three dimensions are shown. In Figure 11a, we start with a triangle/quadrilateral mesh with 1000 entities and generate hierarchies with degrees 2, 3 and 5. Clearly, the results confirm that higher-degree of refinement reach greater resolution much faster. Similarly, in Figure 11b, an initial tetrahderal/hexahedral mesh with 2048 entities is used to generate hierarchies with degrees 2 and 3 . We also observe that the time taken by quadrilateral and hexahedral meshes are more than that taken by trian- gle/tetrahedral meshes. This is difference comes from the data structure updates as quads/hexes have more number of facets to loop over compared to tris/tets. We conclude that if a deep hierarchy is required, a degree 2 refinement per level would give a gradually increasing mesh with more levels. On the other hand, high resolution can be reached very quickly with small hierarchies using high-degree refinement. These serial tests were performed on a Mac computer with $2.3 \mathrm{GHz}$ Intel Core i7 processor and 16GB RAM.

In order to perform the weak scaling studies of the uniform refinement algorithm, we use the Reactor Geometry (and mesh) Generator (RGG [9]) tool developed as part of the generic mesh generation framework MeshKit [23], which encapsulates the workflow of creating a nuclear reactor core geometry and meshes ready for computational analysis in state-of-art physics solvers. Designed with the nuclear engineer in mind, RGG guides the engineer through the process of designing fuel pins, ducts, and assemblies, and then the layout of the reactor core and mesh generation process. We designed a simple unit rectangular lattice based assembly consisting of four fuel pins with six boundary layers around them as shown in Figures $12 \mathrm{a}$ and $12 \mathrm{~b}$. The total assembly generation including the geometry and mesh generation was done only once, which took about 29 seconds. The mesh generation was performed using 


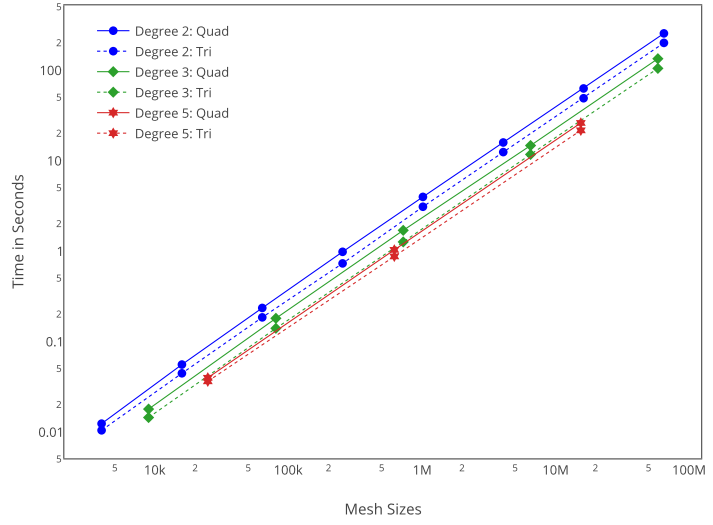

(a) Triangle/Quadrilateral Meshes

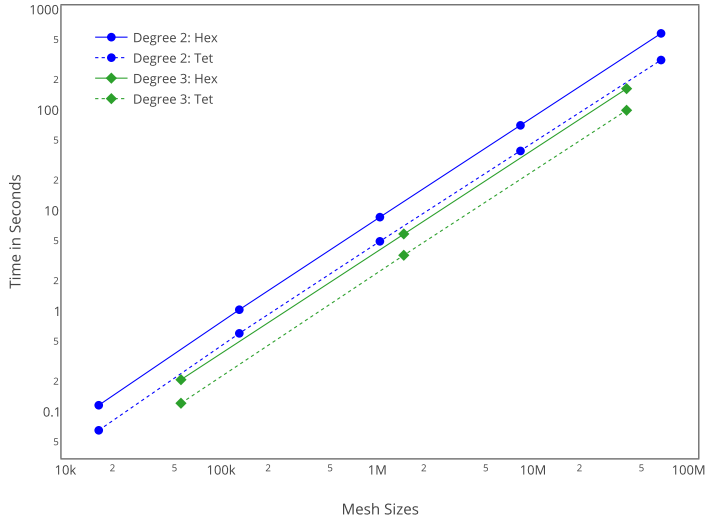

(b) Tetrahedral/Hexahedral Meshes

Figure 11: Time taken to generate hierarchies in serial for different degree of refinements.

Cubit. These unit assemblies were used to create composite assemblies increasing linearly with the number of processors in a pre-decided pattern as shown in Figure $12 \mathrm{c}$ and Figure 12d with two levels of degree 2 of further refinement to increase the resolution. The initial mesh contained about 19,800 hexahedrons with approximately $158 \mathrm{~K}$ and $1.27 \mathrm{M}$ hexes in the subsequent refinement levels. The tests were performed on the Blues cluster at Argonne National Laboratory, which has 310 nodes, 16 cores/node with Intel Sandy Bridge processor and 64GB RAM per node.

The weak scalability results are shown in Figure 13. The merge based shared interface resolution algorithm for UMR scales similar to the ideal case for up to 512 processors. However, we see a drop in the efficiency around 1024 processors which might be due to a number of factors such an uneven load distribution of mesh entities, physical distribution of cores or an overloaded test platform. The time taken to resolve the shared entities for the initial mesh is almost an order of magnitude faster than those taken for the two levels of refinement. This is because the time for shared interface resolution for UMR involves resolution of approximately 4 and 16 times of the initial number of entities on the shared entities. The mesh refinement by itself scales perfectly.

Next, we present preliminary results comparing the merge-based and optimized resolve shared interface algorithms. We use a torus with approximately $2.6 \mathrm{M}$ vertices and 5.3M triangles as an initial mesh with one level of degree-2 refinement. Figure 14 shows an example mesh with eight partitions. Table 4 lists the times taken

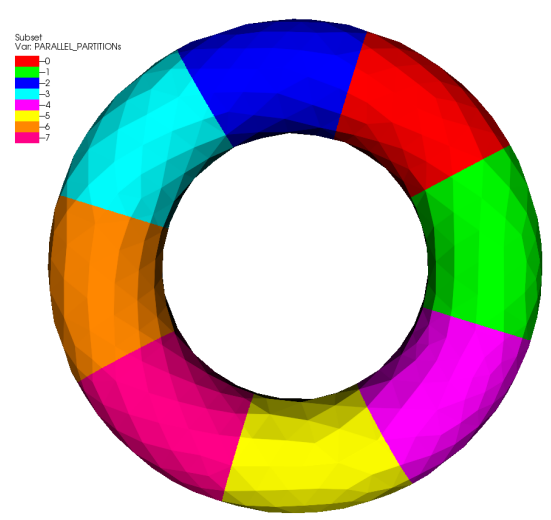

Figure 14: An example torus mesh with eight partitions used for the scalability study of mesh hierarchy generation.

Table 4: The times in seconds for the refinement, resolution of shared entities using the merge and optimized algorithms as the number of processors increases.

\begin{tabular}{|c|c|c|c|}
\cline { 2 - 4 } \multicolumn{1}{c|}{} & \multicolumn{3}{c|}{ Times in Seconds } \\
\hline \#Partitions & Refine & Merge & Optimized \\
\hline 1 & 9.98 & 0 & 0 \\
\hline 2 & 5.31 & 14.13 & 0.078 \\
\hline 4 & 2.45 & 7.4 & 0.063 \\
\hline 8 & 1.3 & 3.8 & 0.073 \\
\hline
\end{tabular}




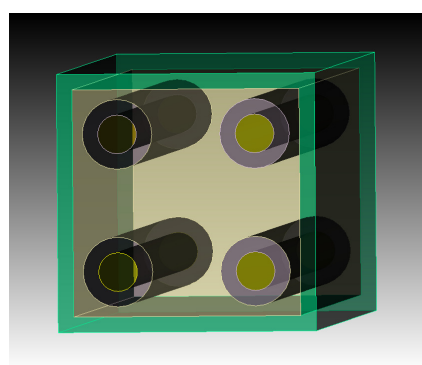

(a) Unit assembly geometry.

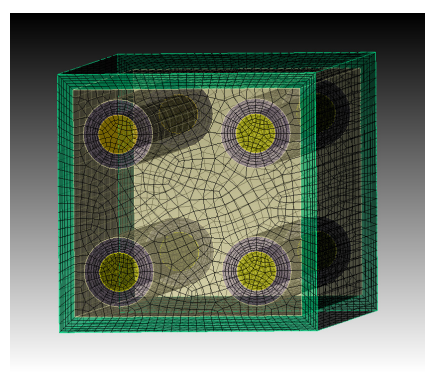

(b) Unit assembly mesh.

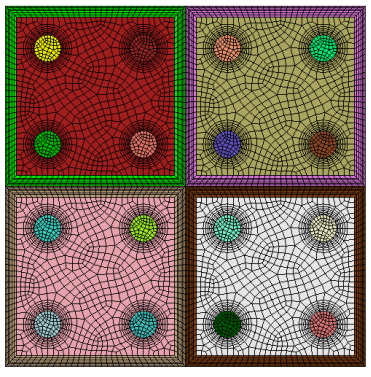

(c) $2 \times 2$ configuration.

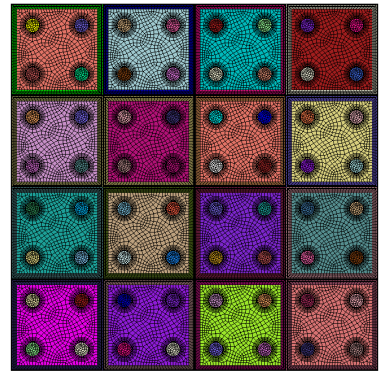

(d) $4 \times 4$ configuration

Figure 12: The initial mesh on each processor and creation of the whole assemblies from the unit.
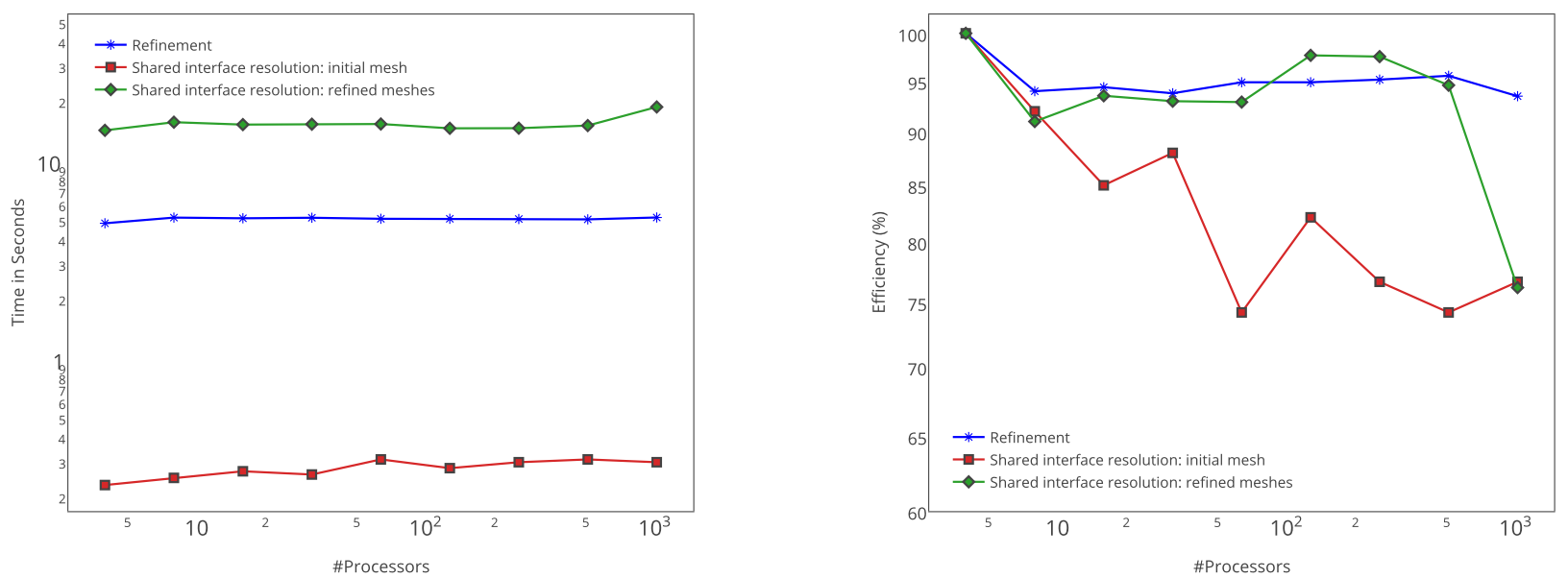

Figure 13: Weak scaling studies for the mesh hierarchy generation algorithm using the RGG tool with two levels of uniform refinement on Blues. The left and right figure shows the time in seconds and efficiency as the number of processors increases with fixed problem size. 
Table 5: Numbers of ghost layers for WALF in parallel.

\begin{tabular}{|c|c|c|c|c|c|c|}
\hline Degree of WALF & 1 & 2 & 3 & 4 & 5 & 6 \\
\hline \hline \#Ghost Layers & 2 & 2 & 3 & 3 & 4 & 4 \\
\hline
\end{tabular}

in seconds by the refinement and the two interface resolution algorithms for up to eight cores. Clearly, the optimized resolve shared algorithm shows a performance improvement of almost two orders of magnitude. However, we see no scaling as the core counts are increased as observed for the merge-based algorithm. The underlying reason behind is that the communication pattern (2 shared processors) and the total number of entities (approx. 2K edges) on the shared interface does not change for these lower core counts. As a result, for the optimized algorithm, the amount of work remains exactly the same compared to the merge-based algorithm. Currently, more rigorous tests are being performed for different distribution patterns on more number of processors on Blues.

\subsection{Boundary Reconstruction}

A key aspect of the refinement algorithm is the positioning of the new vertices as entities are refined. Using a linear point projection scheme for the new vertices would compromise the accuracy of the geometry and in turn that of the finite element solver. To address this issue, we incorporate a polynomial based high-order boundary reconstruction strategy, Weighted Averaging of Local Fittings (WALF), in [11]. WALF constructs third and even higher order accuracy, while guaranteeing $C^{0}$ continuity. Since WALF utilizes local polynomial fittings, it is relatively straight-forward to parallelize. As long as sufficient ghost layers are provided, the reconstruction algorithm performs only local computation without any parallel communication. The required numbers of ghost layers for various degrees of WALF are listed in Table 5. The reconstruction algorithms have been implemented as a submodule, Discrete Geometry, under the framework of MOAB and supports high order surface reconstruction both in serial and parallel.

We first provide numerical results to show that the high-order approximations based point-projection schemes match the theoretical order of convergence under mesh convergence studies over exact analytical surfaces in both serial and parallel. For our experiment, we used a sequence of refined sphere meshes and torus meshes (mesh sizes are listed in Table 6). We randomly generate few points (10) on each element of the mesh
Table 6: Mesh sizes of the sequence of sphere and torus meshes used for convergence studies.

\begin{tabular}{|c|c|c|}
\hline \multirow{4}{*}{ Sphere Meshes } & \#Vertices & \#Triangles \\
\cline { 2 - 3 } & 210 & 416 \\
\cline { 2 - 3 } & 834 & 1664 \\
\cline { 2 - 3 } & 3330 & 6656 \\
\cline { 2 - 3 } & 13314 & 26624 \\
\cline { 2 - 3 } & 53250 & 106496 \\
\cline { 2 - 3 } & 212994 & 425984 \\
\hline
\end{tabular}

\begin{tabular}{|c|c|c|}
\hline \multirow{4}{*}{ Torus Meshes } & 168 & 336 \\
\cline { 2 - 3 } & 672 & 1344 \\
\cline { 2 - 3 } & 2688 & 5376 \\
\cline { 2 - 3 } & 10752 & 21504 \\
\cline { 2 - 3 } & 43008 & 86016 \\
\cline { 2 - 3 } & 172032 & 344064 \\
\hline
\end{tabular}

and then project them onto the high order surface using WALF in order to compute the error introduced by the high-order approximation. The error is computed as the shortest distance from each approximation to the exact geometry.

Figures $15 \mathrm{a}, 15 \mathrm{~b}, 15 \mathrm{c}$ and $15 \mathrm{~d}$ show the $L_{\infty}$ errors of WALF for the sequence of sphere and torus meshes in serial and parallel. In the legend, the degree indicates the degree of polynomial fittings used by WALF, and "linear" indicates the error of linear interpolation. The average convergence rates are shown along the right of the plots, which was calculated as $\log \left(\right.$ error $_{5} /$ error $\left._{0}\right) / \log \left(h_{5} / h_{0}\right)$, where error ${ }_{i}$ denotes the $L_{\infty}$ error on $i$ th mesh and $h_{i}$ is the maximum edge length of the corresponding mesh. We observe that WALF does indeed achieve high order accurate geometry approximation, especially compared with the linear interpolation. In the next section, we discuss how the highorder boundary reconstruction is used to project the new points after refinement and their effect on the accuracy of the numerical solution of the PDE system.

\subsection{Demonstration of UMR Capabilities in Discrete Solvers}

The parallel UMR capability is useful in generating hierarchy of meshes to perform convergence studies and for creating optimal multigrid preconditioners for elliptic PDE solvers. A multigrid Poisson solver written using the PETSc-MOAB (DMMoab) interface that leverages the scalability of both the codes and specifically utilizing UMR is presented here for computing order of accuracy efficiently. Figure 16 shows that the Poisson solver with an inhomogeneous source term in 


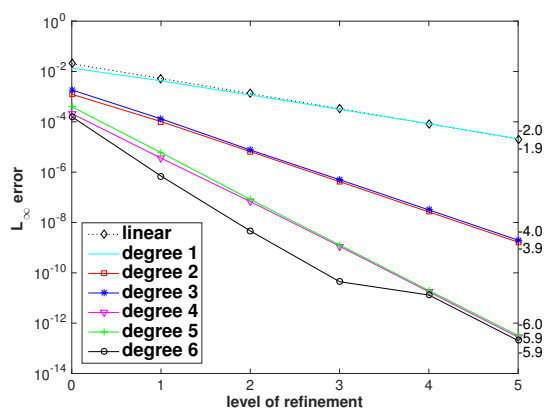

(a) Sphere-Serial

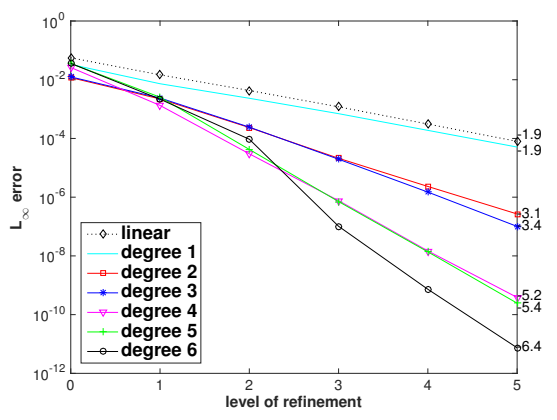

(c) Torus-Serial

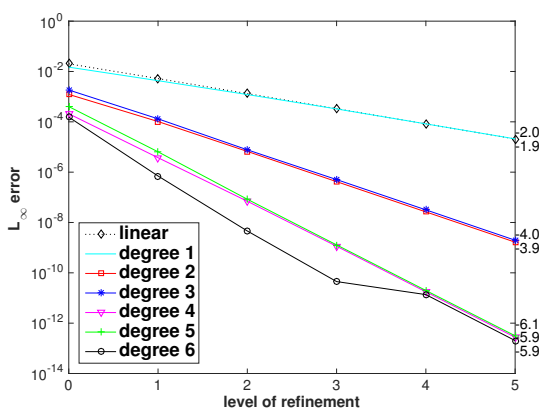

(b) Sphere-8 processors

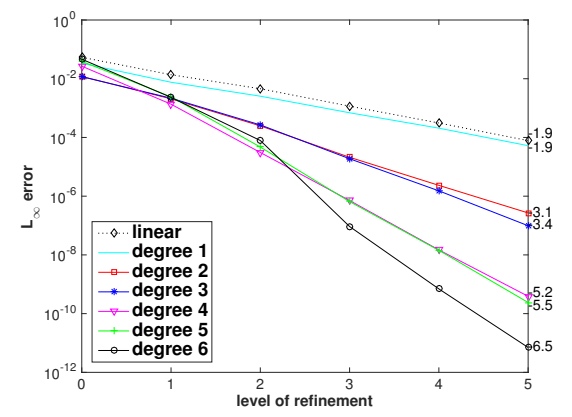

(d) Torus-8 processors

Figure 15: Convergence studies for point projection using high-order surface reconstruction using a sphere and torus mesh in serial and parallel. 


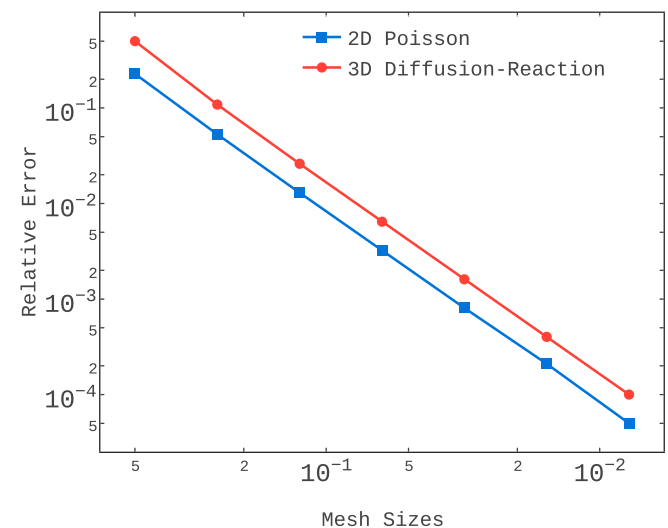

Figure 16: Mesh convergence of a 2D Poisson and 3D DiffusionReaction problem.

2-D and using a Method of Manufactured Solution (sinusoidal exact solution $\sin (\pi x) \sin (\pi y))$ yields the expected second order convergence (linear Lagrange continuous Galerkin FEM). These results were generated by successively refining the mesh through UMR and solved with Geometric Multigrid (GMG).

As a proof of principle, we have performed some preliminary experimental study on the impact of highorder projection on the accuracy of the PDE solutions on curved geometries. We use different high-order projection schemes for the refined meshes and solve the Poisson equation over the approximated curved domain. Only the boundary of the coarsest mesh is reconstructed and used to project the points in subsequent refinement levels as a post-processing step. Clearly, using a highorder approximation reduces the geometrical error significantly, however they remain constant as the order of error introduced by using the reconstructed coarsest mesh does not change. From our experimental studies in 2-D using MATLAB (Figure 17), we have observed significant improvements in the overall accuracy with highorder reconstructions compared to using only piecewise linear reconstructions during mesh refinement. We are conducting more detailed study for 3-D problems in MOAB and will report the results in the future.

In terms of computational efficiency, the iteration convergence of geometric multigrid-based preconditioner for a Poisson solver using a Generalized Finite Difference (GFD) method is also shown in Figure 18 and comparison to standard black-box algebraic preconditioners including AMG, shows optimal reduction

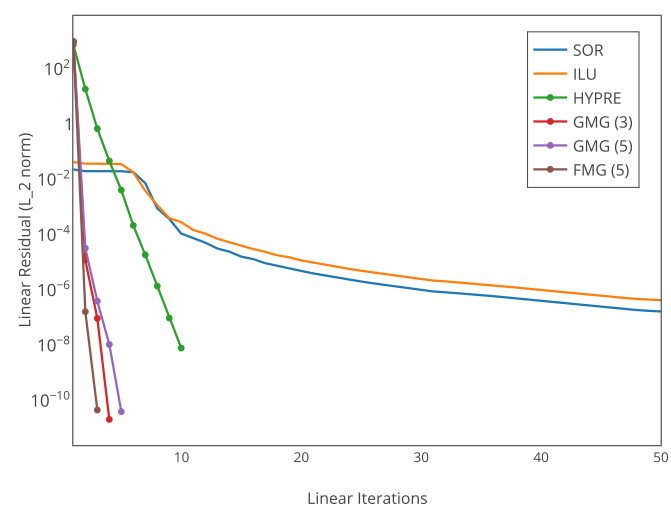

Figure 18: Comparison of convergence of multigrid and standard black-box preconditioners.

in iteration error independent of the mesh resolution or degrees-of-freedom when using Geometric multigrid preconditioners. Figure 18 also shows that GMG with 3 and 5 levels are comparable to a Full Multigrid (FMG) scheme, which is theoretically optimal for solving such elliptic PDE systems.

\section{Conclusion and Discussion}

In this paper, we presented a method for generating a hierarchical unstructured meshes in parallel for efficient solution of PDE's using finite element methods and multigrid solvers. A multi-degree, multi-dimensional and multilevel framework is designed to generate the nested hierarchies from an initial mesh that can be used for a number of purposes such as multi-level methods to generating large meshes. Two parallel communication algorithms are designed to aid in resolution of shared interface. We presented numerical results for computational efficiency of the refinement strategy in a parallel set up as well as the effect on mesh quality. We also demonstrated the applicability of the developed capability for multilevel and finite element methods as well as provide access to different point projection strategies that can effect the solution accuracy.

\section{Acknowledgements}

We thank Cao Lu for his implementation of the GFD Poisson solver with multigrid using DMMoab. We also thank Rajeev Jain for creating and meshing the assemblies for the weak scaling studies. This material is based 


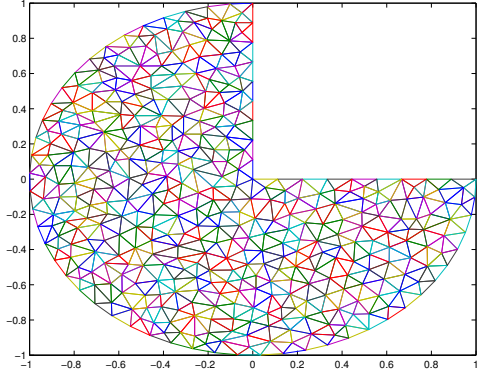

(a) Original mesh

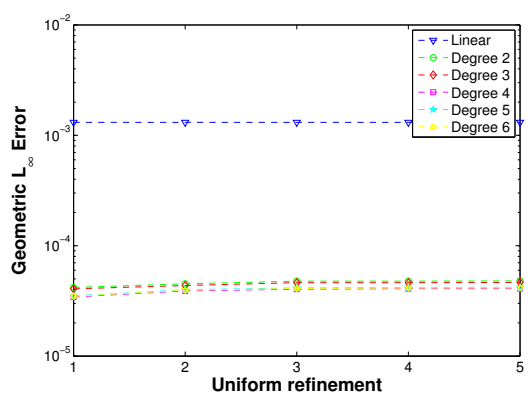

(c) $L_{\infty}$ errors of reconstruction with various degrees of WALF

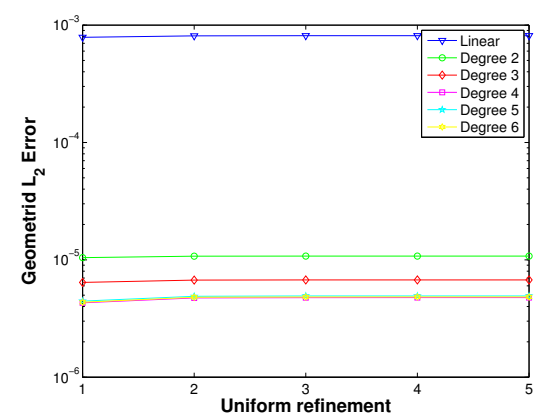

(b) $L_{2}$ errors of reconstruction with various degrees of WALF

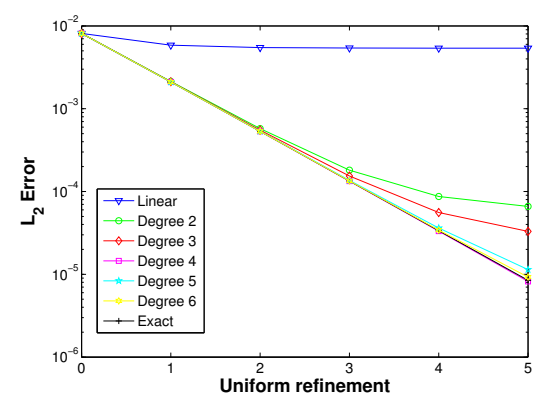

(d) $L_{2}$ error of Poisson equation solution under various reconstructed boundary and exact boundary

Figure 17: Convergence studies for Poisson equation with linear interpolated boundary, WALF reconstructed boundaries and exact boundary 
upon work supported by the U.S. Department of Energy, Office of Science, Office of Advanced Scientific Computing Research, Scientific Discovery through Advanced Computing (SciDAC) program and the FASTMath project. This research used resources of the Argonne Leadership Computing Facility, which is a DOE Office of Science User Facility supported under Contract DE-AC02-06CH11357.

\section{References}

[1] T. Alumbaugh and X. Jiao. Compact array-based mesh data structures. In Proceedings of 14th International Meshing Roundtable, pages 485-504, 2005.

[2] B. S. Bischoff, M. Botsch, S. Steinberg, S. Bischoff, L. Kobbelt, and R. Aachen. OpenMesh - a generic and efficient polygon mesh data structure. In In OpenSG Symposium, 2002.

[3] C. Burstedde, L. C. Wilcox, and O. Ghattas. p4est: Scalable algorithms for parallel adaptive mesh refinement on forests of octrees. SIAM Journal on Scientific Computing, 33(3):11031133, 2011.

[4] L. Diachin, R. Hornung, P. Plassmann, and A. Wissink. Parallel adaptive mesh refinement. Parallel processing for scientific computing, 20:143, 2006.

[5] V. Dyedov, N. Ray, D. Einstein, X. Jiao, and T. Tautges. AHF: Array-based half-facet data structure for mixed-dimensional and non-manifold meshes. In J. Sarrate and M. Staten, editors, Proceedings of the 22nd International Meshing Roundtable, pages 445-464. Springer International Publishing, 2014.

[6] A. Fabri, G.-J. Giezeman, L. Kettner, S. Schirra, and S. Schönherr. On the design of CGAL, a computational geometry algorithms library. Softw. - Pract. Exp., 30:1167-1202, 2000. Special Issue on Discrete Algorithm Engineering.

[7] G. C. Fox, M. A. Johnson, G. Lyzenga, S. W. Otto, J. Salmon, and D. Walker. Solving Problems on Concurrent Processors: 1. General Techniques and Regular Problems. Prentice-Hall, Englewood Cliffs, NJ, 1988. ISBN 13-823022-6 (HB), 13-823469 8 (PB).

[8] R. V. Garimella. MSTK - a flexible infrastructure library for developing mesh based applications. In Proceedings of 13th International Meshing Roundtable, pages 213-220, 2004.

[9] R. Jain and T. J. Tautges. Generating unstructured nuclear reactor core meshes in parallel. Procedia Engineering, 82(0):351 363, 2014. 23rd International Meshing Roundtable (IMR23).

[10] X. Jiao and D. Wang. Reconstructing High-Order Surfaces for Meshing. In S. Shontz, editor, Proceedings of the 19th International Meshing Roundtable, pages 143-160. Springer Berlin Heidelberg, 2010

[11] X. Jiao and D. Wang. Reconstructing high-order surfaces for meshing. Engineering with Computers, 28:361-373, 2012.

[12] L. Kettner. Using generic programming for designing a data structure for polyhedral surfaces. Comput. Geom. Theo. Appl., 13:65-90, 1999.

[13] B. S. Kirk, J. W. Peterson, R. H. Stogner, and G. F. Carey. libMesh: A c++ library for parallel adaptive mesh refinement/coarsening simulations. Engineering with Computers, 22:237-254, 2006

[14] M. Kremer, D. Bommes, and L. Kobbelt. OpenVolumeMesh - a versatile index based data structure for 3D polytopal complexes. Proceedings of 21st International Meshing Roundtable, pages 531-548, 2012

[15] A. Langer, J. Lifflander, P. Miller, K.-C. Pan, L. V. Kale, and
P. Ricker. Scalable algorithms for distributed-memory adaptive mesh refinement. In Computer Architecture and High Performance Computing (SBAC-PAD), 2012 IEEE 24th International Symposium on, pages 100-107. IEEE, 2012.

[16] N. Ray. High-Order Surface Reconstruction and its Applications to Surface Integrals and Surface Remeshing. PhD thesis, Stony Brook University, 2013.

[17] N. Ray, I. Grindeanu, X. Zhao, V. Mahadevan, and X. Jiao. 24th international meshing roundtable array-based hierarchical mesh generation in parallel. Procedia Engineering, 124:291 - 303, 2015.

[18] D. Ren and D. D. Giannacopoulos. Parallel mesh refinement for 3-d finite element electromagnetics with tetrahedra: Strategies for optimizing system communication. IEEE transactions on magnetics, 42(4):1251-1254, 2006.

[19] E. S. Seol. FMDB: Flexible Distributed Mesh Database For Parallel Automated Adaptive Analysis. PhD thesis, Rensselaer Polytechnic Institute, 2005.

[20] D. Sieger and M. Botsch. Design, implementation and evaluation of the surface mesh data structure. In In Proceedings of the 20th International Meshing Roundtable, 2011.

[21] T. Tautges, R. Meyers, and K. Merkley. MOAB: A meshoriented database. Technical report, Sandia National Laboratories, 2004.

[22] T. J. Tautges. Canonical numbering systems for finite-element codes. International Journal for Numerical Methods in Biomedical Engineering, 26(12):1559-1572, 2010.

[23] T. J. Tautges, J. Kraftcheck, J. Porter, A. Caceres, I. Grindeanu, D. Karpeev, R. Jain, H.-J. Kim, S. Cai, S. Jackson, J. Hu, B. Smith, C. Verma, S. Slattery, and P. Wilson. MeshKit: a Open-Source library for mesh generation. In Proceedings, SIAM Conference on Computational Science \& Engineering, Reno, NV, Mar. 2011. SIAM.

[24] T. J. Tautges, J. A. Kraftcheck, N. Bertram, V. Sachdeva, and J. Magerlein. Mesh interface resolution and ghost exchange in a parallel mesh representation. In Proceedings of the 2012 IEEE 26th International Parallel and Distributed Processing Symposium Workshops \& PhD Forum, IPDPSW '12, pages 16701679, Washington, DC, USA, 2012. IEEE Computer Society.

[25] K. Tim and T. Preusser. Stability of the 8-tetrahedra shortestinterior-edge partitioning method. Numerische Mathematik, 109:435-457, 2008.

[26] L.-B. Zhang. A parallel algorithm for adaptive local refinement of tetrahedral meshes using bisection. Numerical Mathematics A Journal of Chinese Universities English Series, 2(1):65-89, Feb 2009.

[27] X. Zhao, R. Conley, N. Ray, V. S. Mahadevan, and X. Jiao. 24th international meshing roundtable conformal and non-conformal adaptive mesh refinement with hierarchical array-based halffacet data structures. Procedia Engineering, 124:304 - 316, 2015 . 